\title{
Narrating the self: Freud, Dennett and complexity theory
}

\section{Tanya de Villiers and Paul Cilliers}

\author{
Department of Philosophy, University of Stellenbosch, \\ Private Bag X1, Matieland, 7602 \\ E-mail:<tdev@sun.ac.za>
}

\begin{abstract}
Adopting a materialist approach to the mind has far reaching implications for many presuppositions regarding the properties of the brain, including those that have traditionally been consigned to "the mental" aspect of human being. One such presupposition is the conception of the disembodied self. In this article we aim to account for the self as a material entity, in that it is wholly the result of the physiological functioning of the embodied brain. Furthermore, we attempt to account for the structure of the self by invoking the logic of the narrative. While our conception of narrative selfhood incorporates the work of both Freud and Dennett, we offer a critique of these two theorists and then proceed to amend their theories by means of complexity theory. We argue that the self can be characterised as a complex system, which allows us to account for the structure of the material self.
\end{abstract}

\section{Introduction}

In the twentieth century the advent of the Freudian conception of the unconscious radically expanded the possible spheres of mental life by doing away with the assumption that the psychical is equivalent to the conscious. Methods such as studying dreams, slips of the tongue etc., and the idea that in some instances, an external observer, like a psychologist, might have more insight into a person's motivations than the person him/herself, opened a wealth of opportunities to rethink many received wisdoms about the mind. In light of his work, many presuppositions that have been taken for granted in the rationalist/empiricist debate on mind, had and still have to be re-evaluated, and an isolationist theory of mind becomes all the more implausible for it.

In contemporary cognitive theory the existence of the unconscious is generally accepted, even while the denotation of the term remains ambiguous. More interesting for the purposes of this paper are the ambiguities that surround consciousness and the lingering tendency to equate the conscious with the immaterial or the metaphysical.

In this paper we would argue that it is entirely plausible and possible to conceive of (and model) the self (as a fundamental aspect of consciousness) as a wholly material entity, and, more specifically, as an emergent property of a complex system; one whose evolution and function is encompassed in the evolution and development of the human brain. When writing on "the self" one's work is considerably complicated by the elusiveness of the phenomenon under discussion. The complication arises from both terminological considerations and the sheer number of phenomena that can 
(mostly justifiably) be lumped together as aspects or instances of the "self". Furthermore, certain presuppositions concerning the kind of phenomena that belongs to this realm have appreciably influenced resultant theories.

Soul, self, consciousness, ego, agent, subject, mind, identity, self-concept and personality are all terms that frequently and significantly overlap when talking about the "higher functions" of the brain. What most of these concepts have in common is their reference to that which "animates" the apparently inanimate matter of which our bodies are comprised - in an attempt to explain that which makes us human; that which allows the capacity for reflection and especially self-reflection, and (apparently) willed action. In this paper, such a capacity is what we are referring to when we use the term "consciousness". With regard to the "self", a sparse definition which could serve us at the outset of our discussion could be something along the lines of describing the self as an aspect of consciousness - the awareness of "me as subject", as opposed to the totality of objects that I am aware of and that are "not-me". Our point of departure could then be that we are examining that "something" that enables us to be aware of ourselves as distinct from the world (the "not-me"), and to actively orient ourselves with regard to that awareness. Furthermore, the self can be defined as human beings' ability, not only to conceive of themselves as agents within the world, but also the ability to some extent to understand and amend their way of being. In other words, the ability not only to conceive of their notions, ideas, motivations, etc., but also to understand that and how they may differ from those of other people, and even the ability to have an idea as to their origins.

Influenced by the groundbreaking work done by Freud, Dennett attempts to account for a theory of self that develops from the material functions of the body, and especially the brain. Dennett's material self takes the form of a "narrative", where the creating and relating of the self is a fundamental human attribute, rooted in our evolutionary history. In this paper, Dennett's materialism will underpin our effort to demystify the self, and to present it as one of the material processes of the brain. Although we agree with his narrative theory of self, Dennett's theory is not without its problems, and we will attempt to address some of these problems through recourse to complexity theory. ${ }^{1}$

\section{Consciousness After Freud}

Freud suspected that, far from being clear and accessible ${ }^{2}$ to detached introspection, consciousness, and by implication rational thought processes, were the result of intricacies of the body's physiological functioning (Freud 1950 [1895]: 307-308). His aim was to establish psychology as a natural science by representing "psychical processes

1 The field studying complex, evolving systems is relatively young (having its origins predominantly in the 1940's and '50's) and vast, incorporating divergent traditional academic disciplines and approaches. Consequently, when working within this discipline one is often faced with a quagmire of possibly confusing and probably overlapping terminology. (See "Classic publications on complex, evolving systems: a citation based survey" by F. Heylighen for a concise overview of some of the main developments, thinkers and publications in this field). "Complexity Theory" for the purposes of this paper should be understood as: "The study of how critically interacting components self-organise to form potentially evolving structures exhibiting a hierarchy of emergent system properties" (Lucas 2003:1). This should be distinguished from "Computational complexity theory" as a subset of the theory of computation. Complexity theory in this sense is defined by the Wikipedia Encyclopaedia (2004) as: "dealing with the resources required during computation to solve a given problem".

2 This is in direct opposition to Descartes' idealistic conception of the mind as lending itself to abstract introspection (Descartes 1978:75). 
as quantitatively determinate states of specifiable material particles" (Freud 1950 [1895]: 295). Consequently he set about modelling the mental apparatus, and providing a physiological description of "mental" phenomena.

He knew that the nervous system consisted of distinct and similarly constructed neurons that have contact with one another through the medium of a "foreign substance" (Freud 1950 [1895]: 298), although he did not know what this substance was. He also knew that certain lines of conduction were laid down between neurons and that they receive and give off excitations. From these basic facts Freud constructed a model of the mental apparatus that would reverberate throughout his later work (Strachey 1986:290) and those of subsequent theorists. ${ }^{3}$

Freud conceives of "neuronal excitation" as quantity ${ }^{4}(\mathrm{Q})$ in a state of flow, and consequently the principle postulates the principle of neuronal inertia: neurons tend to divest themselves of Q. This principle is then used to explain the structure, functions and development of neurons (Freud 1950 [1895]: 296). Crudely simplified, it is this quantity that stimulates the nervous system, and the divestment of quantity through the nervous system to the muscular system, which enables one to react to a state of affairs in the external world. With this model Freud is able to explain stimulus/ response and reflexive behaviour. Discharge of Q is seen as the primary function of the nervous system.

There is, however, another source of energy, which also upsets the principle of inertia and is not so easy to divest. $Q \tilde{n}$ can be explained as a form of endogenous energy. This energy has its origin in the cells of the body itself, especially in the basic needs of the body - hunger, respiration and sexuality ((Freud 1950 [1895]: 297). ${ }^{5}$ Although $Q \tilde{n}$ also obeys the principle of tending towards equilibrium, it cannot be discharged without the body's needs being satisfied. If external conditions are not conducive to meeting bodily needs, this energy cannot be discharged and builds up in the nervous system.

Freud postulates that the accumulation of energy $(Q \tilde{n})$ in the mental apparatus will create an urgency to discharge this energy. The only way for the pressure to be relieved is to affect an external change to get rid of the stimulus that causes the release of $Q \tilde{n}$ in the interior of the body. Initially (in infancy) the human organism is incapable of bringing about the necessary action, and it has to take recourse to external help, in the form of a caretaker. The child's internal state needs to be communicated to the caretaker, which is accomplished "by discharge along the path of internal change" (Freud 1950 [1895]: 318).

Having had his needs met by the helpful external agent, the infant is able to remove the endogenous stimuli and so experience satisfaction. Freud declares that this process

3 He never considered his model to be complete and ultimately abandoned his project due to a lack of physiological knowledge. He did not, however, abandon the belief that such a project is, in principle, possible (Zangwill 1998:277).

4 Freud distinguishes between two "types" of Q:

$\mathrm{Q}=$ Quantity (in general, or in the order of magnitude in the external world), hence where energy is received from the external world through the senses.

$Q \tilde{n}=$ Quantity (of the intercellular order of magnitude); a kind of internal energy.

5 See Freud's Instincts and their Vicissitudes (1915a: 117-140) where he elaborates on this basic scheme and establishes instinctual stimuli as stimuli arising from the organism itself, operating as constant forces. 
has a radical influence on the development of the individual's functions. ${ }^{6}$ Contact barriers are facilitated between neurons when energy is discharged due to needs being met. $Q \tilde{n}$ passes more easily between facilitated neurons than between ones that are not facilitated. These contact barriers ensure that consciousness would move from a stimulated neuron to one that had previously also been stimulated in similar circumstances. When a certain urgency is experienced once again, the relevant memories are triggered. ${ }^{7}$ Motor discharge is now allotted with the function of action, in other words with the task of altering reality (Freud 1950 (1895): 307-340). As such Freud presents memory as the basic component of the nervous system and as prior to consciousness and cognition. The procedure of forming memory traces is entirely unconscious, with the capacity for becoming conscious, and can be influential while being in an unconscious state (Freud 1901:539).

Freud recognises the immense implication that his theories on the unconscious would have on theories of mind. ${ }^{8}$ Whereas, before the advent of the unconscious, "psychical" was considered to be equivalent to "conscious", complex psychical processes now become possible that do not rise to consciousness and, in fact, the unconscious becomes the basis and the larger sphere of mental life. As Freud insists: "It is essential to abandon the overvaluation of the property of becoming conscious before it becomes possible to form any correct view of the origin of what is mental" (Freud 1901:612; see also 1923:13).

After Freud, no examination of the mind could be complete without taking the unconscious into account. The possibility of phenomena like repression and resistance stemming from the unconscious, and having a compelling influence no matter the will of the subject, paved the way for a dynamical theory mind, where mental forces can be in conflict with one another (See Strachey 1986:19 and Sternberg 1995: 598). Not only did consciousness become dynamic, but it became time-dependent - always already influenced by the traces of memories of past experiences that make up the unconscious.

Unbeknown to him, Freud's model of the mental apparatus in his Project for a Scientific Psychology is an example of a classic complex system, ${ }^{9}$ and his work makes an invaluable step towards a position that allows us to conceive of the "higher functions" of the brain, and the self in particular, as emergent properties of a complex, open and distributed system.

Continuing in the Freudian tradition Daniel Dennett focuses his attention on the dynamic and time-dependent aspect of the mental apparatus in his own attempt to study consciousness as a purely materialistic phenomenon. Important for the purposes of this paper is that he develops his materialistic theory of consciousness into a conception of the self as a materialistic and evolutionary phenomenon. An overview and critique of his theory will serve as an introduction to a proposal for a model of the self, based on complexity theory.

6 The meaning that Freud attaches to this initial and inevitable encounter suggests that social interaction is a necessary precondition for the development of consciousness. See also section VII of The Interpretation of Dreams (1901).

7 Freud returns to this topic again in The Interpretation of Dreams (1901), Beyond the Pleasure Principle (1920), and A Note on the "Mystic Writing-Pad" (1925).

8 See the Unconscious (Freud 1915b:166-171) where Freud defends his view that the postulation of the unconscious is both necessary and legitimate.

9 See Cilliers and Gouws (2001:237-256) for an extended argument supporting a similar conclusion. 


\section{Dennett on the Origin of Mind}

In Consciousness Explained Dennett (1991) differentiates between different phenomena that make up what we call "consciousness," and holds (as did the Freud of The Project) that all of these phenomena are the result of the physical activities of the brain (Dennett 1991:16). ${ }^{10} \mathrm{He}$ insists that the mind is the brain and that all instances of "evidence" to the contrary are illusions created by the properties of these brain processes. ${ }^{11}$ Dennett believes that consciousness arose because the brain "[had] to become the object of its own perceptual systems" (Dennett 1991:225). ${ }^{12}$ In short, the mind (specifically the human mind) arose as a result of the evolution of the human brain in the presence of innumerable environmental pressures. ${ }^{13}$

Dennett further believes that our concept of the self arose as a result of a peculiarity of our minds: they are "witnessed" or experienced. As he puts it: "You know you have a brain the way you know you have a spleen: by hearsay. You've never seen your spleen or your brain ... but since the textbooks tell you that all normal human beings have one of each, you conclude that you almost certainly have one as well. You are more intimately acquainted with your mind - so intimately that you might even say you are your mind" (Dennett 1996:3). And at first blush, the brain does not seem to be anything akin to what we would imagine a witness to be. Hence the idea arose of a self (or soul, or ego, or person) as distinct from a brain or a body. Dennett argues that the observation that where there is a mind, there is a point of view (Dennett 1996:101), need not lead to the conclusion that the mind is distinct from the body, and he sets about exploring the possibilities of the material mind.

The mind-body distinction breaks down for Dennett when he tries to pinpoint a point of consciousness within the brain. He dubs the view that some such central observer exists within the brain Cartesian materialism. Cartesian materialism postulates the existence of a Cartesian Theatre in the brain, a point within the brain, which is the locus of all that we are conscious of (Dennett 1996:107). In Consciousness Explained (1991) he makes a compelling case for the fact that such a point simply does not exist.

10 Dennett's theories in Consciousness Explained (Dennett 1991) are very much influenced by his research on cognitive science. His naturalistic approach here is the result of this research, which has lead him to recapitulate his conception of the basic mental structure twice (cf. Dennett 1994:236).

11 Dennett uses the terms "mind" and "consciousness" relatively interchangeably. What is at issue here is human consciousness/mind as that aspect of the world that we can distinguish from everything else through the way that we know it; an aspect of human phenomenology that arises from "a different kind of knowledge" (Dennett 1996:2). To Dennett a conscious being has agency, but it also needs to have an understanding of meaning, in other words, it needs to exhibit intentionality. Dennett subscribes to a Darwinian intentionality; an intentionality that developed over billions of years from "the awkward, ugly period of featherless pseudo-intentionality" of organisms to the human mind, which is intentional (Dennett 1995:205-206). He proposes that we treat all human beings as intentional beings, because we share a subjective world, and know we do, by means of language (or the capacity for language) (Dennett 1996:9). We will elaborate on these ideas in the following section.

12 Dennett's theory echoes that of Freud as discussed above. For Freud, consciousness arises when the human organism needs to alter external conditions to meet internal needs (the discharge of stimuli in the mental apparatus). In order for this alteration to take place it needs to be able to assess and interpret those conditions - it needs what Freud calls "the reality principle".

13 In Kinds of Minds Dennett argues that human minds are very different from nonhuman animal minds (Dennett 1996: 12-18), primarily because of language. These ideas will be elaborated on in the following section. 
${ }^{14}$ He concludes that the brain is the "headquarters" of the perceived observer, and that there is no other, deeper headquarters in the brain where consciousness is seated.

A brain without a Cartesian Theatre significantly complicates the concept of the self. If there is no "consciousness centre" in the brain, which, when stimuli reach it, causes them to become "known" to us, the observer's subjective sense of experience must then be determined by something other than "order of arrival" of experienced items to the "seat of consciousness" in the brain (Dennett 1996:107). In Consciousness Explained Dennett proposes the Multiple Drafts version of how consciousness comes about, to replace the concept of the Cartesian Theatre (Dennett 1991:101-170). The Multiple Drafts model holds that, in his words: "all varieties of perception - indeed, all varieties of thought, of mental activity - are accomplished in the brain by parallel, multi-track processes of interpretation and elaboration of sensory inputs" (Dennett 1991:111).

In fact, Dennett argues, it is misleading to ask the question of when perceptions become conscious; the information content gleaned from sensory inputs is distributed throughout different systems of the brain. These, what he calls, "distributed contentdiscriminations" become something like a narrative stream - a multiplicity subject to continual editing by many processes distributed throughout the brain (Dennett 1991: 113). There is no single, final narrative that can be considered to be the actual stream of consciousness of the subject. As he notes, some contentful (sic.) states might die out completely, leaving no trace, while others do leave traces that might later arise in some form or another, for example a verbal report, or an emotional state (Dennett 1991: $135) .^{15}$

Dennett's proposed Multiple Draft model of the conscious processes within the brain allows him to rethink and recast the self. With no single stream of consciousness, and no central centre of command in the brain, there cannot be a "grand self" overseeing or even witnessing the surrounding goings on. And yet, within the multiple channels within the "virtual machine" of the brain, and within the various narrative drafts that may or may not play functional roles in the activities of the brain it is impossible to deny that human being do have a (relatively?) coherent sense of self.

It seems to Dennett to be a remarkable fact of nature that each member of the primate Homo sapiens constructs a self (Dennett 1991:416). Dennett refers to the self as "a web of words and deeds" and likens the inclination on the part of human beings to construct a self to the inclinations of spiders to spin webs or bowerbirds to construct elaborate bowers - a self is crucial to our success as human beings (Dennett 1991: 416). Dennett also uses the image of the beaver constructing a protective fortress through actively gathering the necessarily material and sharing the labour among individuals of the species. In a like manner, humans appropriate all manner of "found" cultural objects, with which, among other things, to construct their own web of self.

14 Dennett's objection against the Cartesian Theatre is concisely summed up in the following quote:

The Cartesian Theatre may be a comforting image because it preserves the reality/appearance distinction at the heart of human subjectivity, but as well as being scientifically unmotivated, this is metaphysically dubious, because it creates the bizarre category of the objectively subjective - the way things actually, objectively seem to you, even if they don't seem that way to you! (Smullyan 1981, quoted in Dennett 1991:132).

15 With regard to our discussion on consciousness it is interesting to note that not all information recorded by the body's receptors is passed on to the higher levels of the central nervous system; processing of the data already begins at the sense organ (cf. Nathan 1998:515). In a similar vein, all the information reported by receptors does not necessarily reach consciousness (Nathan 1998:515). 
These found objects are the objects of culture. In Darwin's Dangerous Idea Dennett states his case more vehemently: "What we are is very much a matter of what culture has made us" (1995:340).

Human beings are constantly engaged in presenting themselves to other human beings and to themselves, through the ubiquitous medium of language. We tell stories of who we are, where we come from and where we are going - the result is what Dennett calls this construct our "narrative selfhood" (Dennett 1991:418).

\section{Self-ish memes}

As Dennett notes, one definite advantage of the Multiple Drafts model is that it lends itself to a theory of the evolution of consciousness (Dennett 1991:171-266). Far from proposing a metaphysical, non-bodily phenomenon, the Multiple Drafts model leads to a picture of a Darwinian consciousness, which developed with a species, in accordance with constraints and possibilities imposed by that species' environment and genetic adaptations.

Dennett presents the design of human conscious minds as the result of three evolutionary processes (Dennett 1991:173) where the need for self-preservation and control, through the ability to track and anticipate events, gave rise to the development of the human nervous system in successive guises. In terms of evolutionary theory, systems proficient in information gathering, and geared towards information that is beneficial to the organism, develop - these systems become part of the innate design of the nervous system. Of course, information gathering and assessment need not necessarily occur in a conscious state.

Dennett goes on to speculate that the development of nervous systems that possess an element of plasticity, and that therefore have the ability to learn in the course of their lifetime, provided other (other than genetic) media in which the evolution of such nervous systems could occur. This allowed the evolutionary process, hitherto driven by natural selection and genetic mutation, to speed up (Dennett 1991:182). Such a learning mechanism would operate along the same lines as "natural" (Darwinian) evolution, in other words, a process of evolution through selection. Dennett refers to this particular kind of evolutionary process as post-natal design fixing (Dennett 1991:183). It could roughly be characterised as a process of learning rather than development. ${ }^{16}$

A plastic, adaptable brain (the cortex) is the first "new" medium in which the evolutionary process with regard to nervous systems can be sped up. In fact, Dennett attributes the radical transformation of human society in the last 10,000 years (the development of agriculture, art, cooking, etc.) to new ways our ancestors developed to harness "new" mental capabilities. He uses the metaphor of creating software, which could be run on the wired-in hardware of the Homo sapiens brain (Dennett 1991:190).

In our brains there is a cobbled-together collection of specialist brain circuits, which, thanks to a family of habits inculcated partly by culture and partly by individual self-exploration, conspire together to produce a more or less orderly, more or less effective, more or less well-designed virtual machine... By yoking these independently evolved specialist organs together in common cause, and thereby giving their union vastly enhanced powers, this virtual machine, this software of the brain, performs a sort of internal political miracle: It creates a virtual captain of the crew, without elevating any one of them to long-term dictatorial power. Who's in charge? First one coalition and then another, shifting

16 This distinction is by no means clear, but for simplicity's sake we shall refer to post-natal design fixing as "learning". 
in ways that are not chaotic thanks to good meta-habits that tend to entrain coherent, purposeful sequences rather than an interminable helter-skelter power grab $(1991: 228){ }^{17}$

The second new type of evolution that Dennett discusses, is cultural evolution (Dennett 1991:193). This evolutionary medium is the product of the plasticity of the brain, which makes learning possible. Through cultural transmission we install already developed "programmes" of behaviour in developing (usually young) minds. Dennett calls this process of relating information "software-sharing", and it happens, of course, through some or other form of language (Dennett 1991:194; 1995:347). Through honing the art of software-sharing, culture develops into what Dennett calls "a repository and transmission medium for innovations" (Dennett 1991:199).

The final new evolutionary medium that Dennett discusses is the medium through which cultural products are transmitted from nervous system to nervous system: memes (Dennett 1991:199; 1995:341). ${ }^{18}$ Memes are the smallest unit of recognisable information that can be transmitted between brains. They implement themselves in the human nervous system through cultural transmission and so affect changes within this system. Memes operate within the infosphere, which is created by human language (Dennett 1995:347). Speaking, hearing, writing, and reading are the underlying technologies of replication in the infosphere and analogous to RNA and DNA in the biosphere. ${ }^{19}$ In fact, Dennett (Dennett 1991:210) describes human consciousness as "a huge complex of memes". The "narrative selfhood" that makes up the self that the human organism presents to itself and to the world would be made up of memes.

We have seen that Dennett describes the self as a narrative selfhood that the active body includes in a mental model of itself in its representation of its environment, in order to facilitate long-term planning. By having a model of itself the body can also keep track of its internal states, decisions, tendencies, etc. (Dennett 1991:427-428). But, taken in this light Dennett's recourse to memes is one of the weakest aspects of his theory. ${ }^{20}$ The main (and perhaps most damning) criticism that can be levelled at Dennett's use of memes in this regard is that he seems to lose much of the ground that he gained by expelling the homunculus, in the guise of the Cartesian Theatre, from his theory on mind. His memes seem to take on a mind of their own, in that they, like genes, are driven to replicate themselves, which they do by gaining access to the human mind.

17 In a similar vein in The Interpretation of Dreams (Dennett 1901:610) Freud is careful to caution his readers against assigning a topographical locality to the mental apparatus. What he has in mind with his theory on mental functioning is a dynamic process, where a particular mental grouping has a "cathexis of energy" attached or withdrawn from it, and thus comes under the sway of an agency. These systems are not physical entities and are described as being virtual - existing between elements of the organic nervous system "where resistances and facilitations provide the corresponding correlates" (Dennett 1901:611).

18 See Dawkins (1976) where he develops his theory of memes. Memes follow the same basic evolutionary principles as the genes of Dawkins's theory in The Selfish Gene, where Dawkins proposes that Darwinian natural selection does not take place between individual organisms, but, in fact, takes place between genes. Dawkins's view is highly controversial. Gould, for example, describes this "hyper-Darwinian" idea as a "logically flawed and basically foolish caricature of Darwin's genuinely radical intent" (Dawkins 2000:87).

19 Dennett does not take this analogy to be straightforward: he discusses similarities between memes and genes (Dennett 1995:342-352) as well as dissimilarities (Dennett 352-360).

20 It is perhaps telling that memes are omitted entirely in his later work Kinds of Minds, where Dennett's ideas on the evolution and formation of mind are refined. 
Ultimately the idea of consciousness as a complex of memes, loses the idea of consciousness as part of the human body and born of its interests. It is hard to read Dennett on memes and not form a conception of memes acting entirely in, and using the human body for, their own interests. Dennett seems to be aware of this possibility and tries to counter it with his assertion that the memes that end up being replicated, that become part of culture and consciousness in other words, are those that are "good for us".

Taking a memetic perspective on culture leads to the implication that "a cultural trait may have evolved in the way that it has simply because it is advantageous to itself" (Dennett 1995:362). If this is a legitimate way to think about ideas Dennett thinks it is justified to apply meme-theory to culture (Dennett 1995:362). After deliberation he comes to the conclusion that the memes that tend to replicate and hence survive are those memes that tend to be good for us. In other words in doing what is good "for it", replicating itself by manifesting itself in a human body, memes are good for us as well. Such memes are not necessarily good for our biological fitness, but they are good for our "highest values" (Dennett 1995:364). Dennett comes to this conclusion, because he believes, as a crude rule of thumb, that good memes will tend to be good replicators. He dismisses the "memes versus us" polarity, by asserting that our minds are already formed by earlier infestations of memes, and that these infestations play a large part in determining which other memes will find manifestation in the mind: "our selves have been created out of the interplay of memes exploiting and redirecting the machinery that Mother nature has given us" (Dennett 1995:367). Generally, only memes that are good for the self that has already been formed will be allowed "entry" into the mind. In so dismissing the polarity between mind and memes (or in one formulation, self and memes), Dennett does nothing to convince us that the memes that do find manifestation in the mind are by and large "good for us". It is not at all convincing that, generally, the memes that are good for us are the memes that manage to replicate. One might be able to make a case for the role of parenting in this regard, where parents shield the infant mind from "bad" memes and thus ensure a basic blueprint, which regulates future meme intake, but such a scenario seems no more than a caricature of the complexities that would be involved in such a process. And it is all too easy to indicate many instances where people have internalised ideas that aren't good for them; the body-image of an anorexic, for example. Since the compatibility between memes that replicate for their own sake and the manifestation in our brains of memes that are good for us was the test that Dennett proposes for the acceptability of meme-theory as a means of explaining cultural evolution, his ideas seem better served in dropping meme-theory altogether.

\section{Different minds?}

In Kinds of Minds (1996) Dennett revisits and reworks many of the themes and ideas of both Consciousness Explained and Darwin's Dangerous Idea. Although Dennett does not address the matter, it seems significant that memes are not mentioned at all in this work. Instead, he revisits his conception of mind and places much emphasis on language (and the acquisition thereof), as absolutely central to the human mind:

One prospect to consider ... is that perhaps language is not so peripheral to minds after all. Perhaps the kind of mind that you get when you add language to it is so different from the kind of mind that you have without language, that calling them both minds is a mistake. Perhaps, in other words, our sense that 
there are riches in the minds of other creatures - riches inaccessible to us but not, of course, to them - is an illusion (Dennet 1996:17).

In raising the possibility that the "minds" of other animals are nothing like our minds Dennett of course raises the question: why not? In what way do non-human minds differ from human minds? And by the same token, why do we have a tradition of assuming that the minds of some animals, at least, are rather similar to ours? He then goes on to make a case for the proposition that our minds evolved to their present form, primarily because of language.

Dennett raises the possibility that instances of what we call mind could, in fact, be divided according to some sort of scale, distinguishing "classes of pseudominds, or protominds, or semiminds, or hemi-semi-demi-minds" from "real" minds (Dennet 1996:18). A useful way to approach such a task is through the intentional systems approach.

It seems quite obvious that atoms and molecules do not have minds. Yet, DNA and RNA macromolecules have the ability to mindlessly interact in such a way as to replicate themselves - they have agency. These macromolecules (molecular robots as Dennett calls them) are complex enough to have agency (a quasi-agency if you like), but it remains a mindless agency; it seems safe to assume that they are not aware of themselves or their own actions in any way. And, from an evolutionary perspective we, and our minds, are just some of the descendents of such mindless, self-replicating macromolecules (Dennet 1996:19-26).

Our macromolecular ancestors evolved into all living things that inhabit the planet, organising themselves into bodies that need to regulate and protect themselves. Their actions aimed at achieving such self-regulation and self-protection seem remarkably like intentional actions - effects produced by "information-modulated, goal-seeking systems" (Dennet 1996:26). Dennett calls all entities that produce such effects intentional systems, ${ }^{21}$ and suggests that, in studying such entities, we adopt the intentional stance towards them - interpreting their behaviour as if it were the behaviour of a rational agent (Dennet 1996:26-27). Adopting the intentional stance and treating the entity in question as if it were a rational agent seeking its own good is a shortcut that enables one to explain and predict its behaviour. In other words, it is a simple strategy of interpretation that enables one to limit the number of possible causes of a specific effect - one only considers the "smart" moves that are in the "agent's" own best interest. By adopting such an "as-if" intentional stance to all entities that can be characterised as "information-modulated, goal-seeking systems", Dennett is able to include self-replicating macromolecules, thermostats, plants, bats, people and chess-playing computers under the denotation of "intentional system" (Dennet 1996:34). He sees this as the key to unravelling the mysteries of mind(s).

For Dennett the brain is "fundamentally an anticipator, [and] expectation-generator" (Dennet 1996:57), where present and past experience are used to create anticipations of the future. Such anticipations then guide its behaviour, and in so doing contribute to its survival. Earlier or simpler versions of (quasi) intentional systems do not have the capacity to anticipate the future; they do not "appreciate" their own quest for the resources that ensure their livelihood. In other words, they don't need brains.

Dennett (Dennett 1996:81-93) proposes an idealised version of how brains evolved, which he calls the Tower of Generate-and-Test. The ground floor of this tower is made

21 "Intentional" in this sense should be understood as "aboutness", rather than in the more familiar sense of doing something with forethought. 
up of Darwinian creatures - early evolutionary creatures and entirely hard-wired at birth. As the evolutionary process continued, organisms developed with phenotypic plasticity. These creatures make up the next level of Dennett's Tower. He calls them Skinnerian creatures, because of their ability to be conditioned by their environment, as an extension of natural selection. Skinnerian organisms could adjust elements of their designs by events that they experience. By getting positive or a negative feedback from the environment, actions that are "good for them" would be reinforced, thus increasing the likelihood that they would repeat those specific actions in the future. Most animals learn in this manner. Being hardwired has many advantages - the design is robust, fault-tolerant and easy to redesign as it becomes necessary. However, Dennett highlights some cognitive tricks that the Skinnerian design does not allow for. A very useful cognitive skill is that of "one shot learning" or the ability to preselect among possible behaviours. The advantage here is, of course, that an organism does not run the risk of being killed before it can learn from its mistakes through negative reinforcement. Dennett names the organisms that are able to preselect actions, without having to learn through positive or negative reinforcement, Popperian creatures and they make up the third floor of the Tower.

For preselection to take place, Popperian creatures must have some sort of inner environment, where trial runs can safely be performed and assessed. For the assessment to be accurate, the inner environment would need accurate information about the outer environment. Accurate information needs either acquired or inherited information. Information is acquired through various mechanisms for perception. Human beings, but also many animals, are Popperian creatures at some level.

The successors to Popperian creatures are Gregorian creatures (Dennett 1996:99). Gregorian creatures are creatures "whose inner environments are informed by the designed portions of the outer environment" (Dennett 1996:99). These creatures do not only create artefacts, they also gain intelligence from artefacts in that it presents the Gregorian creature with an example of a "Smart Move." The information embodied in the tool need not only be applied to recreate and expand upon its own design, it can also potentially be applied to other situations (Dennett 1996:99). Dennett believes that this ability to generalise is limited, to different degrees, in animals; that is all animals, except human beings (Dennett 1996:100-117). Obviously Dennett's argument is that human beings are Gregorian creatures, and as such our minds are shaped with the tools that we create. One such tool is language. By the same token, given that our minds seemingly are the only minds shaped by tools (except perhaps those of chimpanzees to a limited extent), ${ }^{22}$ it is inappropriate to want to attribute functions of the human mind to animal minds. Hence Dennett's proposal, as we discussed earlier, that we envision a continuum of "mind", which would make it possible to differentiate between specific categories of mind. Dennett sees the development of the ability to adopt the intentional stance towards others as the breakthrough in the animal mind. He discusses possible states of circumstances that would force such a way of thinking to evolve (Dennett 1996:120). The conclusion is that in order for the ability to think in terms of intentionality to emerge, there are a few evolutionary conditions that need to be satis-

22 It is relatively well-known that some chimpanzee groups in the wild have hit upon the idea to use sticks as tools for catching termites, by thrusting it down a termite hole and then swiftly drawing it out crawling with termites to snack on. Meanwhile there are other chimpanzee groups that have not come up with this or any other idea that involves tool-use for catching food. The Smart Move is only available to those who are exposed to it within their particular group (1996:99-100). 
fied. Firstly, the behavioural environment needs to be sufficiently complex; secondly, the capacity for secret-keeping, enhanced by the complex environment, must develop within an organism. The significance of secret-keeping lies in the advantage an agent can gain from hiding valuable resources or other ecologically important information from rival agents, turning the knowledge to its own advantage. Thirdly, as a result of secret-keeping, the ability to communicate must develop. With communication agents can attribute intentionality, both to themselves and rival agents, which would facilitate the discovery of the best manner in which to keep and expand the competitive advantage gained from secrets. ${ }^{23}$ Where these conditions are lacking, it does not become necessary to form a representation of one's own motivations, nor those of one's rivals.

Human beings have the added ability to re-represent their representations, not only in the mind, but also in the environment. We make tools to think with. Such supplementary devices vastly enhance and expand our cognitive abilities (they also serve to transfer information to others). Off-loading onto the environment not only facilitates memory, it also enables us to do cognitive tasks that we would otherwise be unable to do (model making, for example). Without such enhancements we would not be able to pose and contemplate metaphysical questions. We keep much of our information in the external world - in libraries, notebooks, and other people, for example. And the most important mind-tools are, of course, words. Dennett is careful to emphasise that spoken and written language are two separate inventions, separated by hundreds of thousands, if not millions of years, and he contemplates the importance of spoken language in enhancing our cognitive capabilities (Dennett 1996:119-146). He suggests that in learning to label objects and situations with the appropriate words, children acquire the habit of representing their own states and behaviours to themselves in a similar way. Words are the prototypes of concept and as such redesign the "naked human mind" (which, Dennett points out, is something we have never seen) (Dennett 1996:153). Words are multi-purpose tools that can be re-applied in many modes:

Once we have created the labels and acquired the habit of attaching them to experienced circumstances, we created a new class of objects that can themselves become the objects of all pattern-recognition machinery, association-building machinery, and so forth (Dennett 1996:151).

As in Consciousness Explained and Darwin's Dangerous Idea Dennett concludes that mental contents do not become conscious upon entering some "point of consciousness" within the brain, nor by being transduced to some "privileged medium" but by beating other mental contents in the battle for domination in controlling behaviour, in other words, being set down as memories. As he puts it: "What you are, is the agent whose life you can tell about. You can tell us, and you can tell yourself" (Dennett 1996:156).

So, in Kinds of Minds, Dennett again argues for an understanding of the self as a narrative. The self here is not the result of an infestation of memes, nor a vague result of an organism's need to factor itself into its model of the world. The self here arises because of human beings hitting upon the Smart Move of taking up the intentional stance towards other entities in order to more accurately predict their movements and actions. As Dennett sees it, the self is a result of a human organism presenting its own actions and motivations to itself in terms of the intentional stance.

23 This is a very brief overview of Dennett's supposition. See Chapter 5 of Kinds of Minds (1996) for a more comprehensive discussion. 
Dennett's analysis raises many interesting ideas that could lead to much fruitful discussion. Unfortunately limited space restricts us to examine only a few of the possible implications.

\section{Complexity Theory and the Brain - Emergence}

As with the Freud of the Project and Dennett, an approach to the study of brain and consciousness informed by the complexity sciences is materialistic. In this regard an understanding of how the brain (and specifically the cortex) goes about its task is more important than one would think. We know something about neurons and something about psychology, and it is in the gap in between that the enigma of consciousness and the self lies.

As with Dennett's argument, this materialist approach works with the assumption that the higher functions of the brain are grounded in the physiology of the brain. We argue that the structure of the brain lends itself to being modelled as a complex, distributed system and that consciousness is an emergent property of the brain. We will develop the argument that the self, in particular, can be described as an emergent property of the interaction between the physiological processes of the brain and the environment (especially the cultural environment). Dennett's theory on the narrative self and the origins of that self may be enhanced when approached through the perspective of complexity theory.

Instead of approaching a complex system in a reductionistic fashion (as classical physics might do) complexity theory tries to take the relational character of the components of a system into account. Such relational characteristics are liable to be overlooked or even undermined when a system is analysed in a reductionistic manner. Furthermore, a complex system is an open system and, as such, is in continuous interaction with its environment - a relation that also needs to be accounted for. A model of such a system will inevitably reduce the complexity in order to be intelligible (unless the model is a complete copy of the system in question). A complex approach acknowledges this and tries to factor it into the eventual model. The following is a short summary of the characteristics that a system needs to possess in order to be considered a complex, distributed system: ${ }^{24}$

Complex systems are open systems that are made up of many different components. The characteristics of the system are the result of the interaction between these components amongst one another and with the environment. Interactions are generally nonlinear. Furthermore, characteristics that appear complex and disjointed at a relatively small scale generally prove to form recognisable and stable patterns at a larger scale.

A complex system has more possibilities than can ever be actualised. The number of components that can be considered to be a part of a complex system and the number of possible interactions between these different components make for a vast amount of possible configurations and possible future states of the system. As the system increases in size the number of possibilities increases accordingly.

Complex systems have a memory, in that they are systems that evolve through time and that their histories shape them, and in so doing play a major part in their present structures and behaviours. ${ }^{25}$

The concept of emergence is central to theories of complexity. The argument is that there is more to a certain system than is evident from examining the individual parts

24 See Cilliers (1998) for more detail.

25 Complex systems need not be biologically "alive". Language, for example, is a complex system. 
that make up the system. This something "more" is what Morowitz calls "novelties that follow from the system rules but cannot be predicted from the properties of the components that make up the system" (Morowitz 2002:13). In other words, emergent properties are the characteristics of the system that result from the non-linear interactions between the components of the system, amongst themselves and with their environment. Emergence belongs to the structural aspect of the system and systems do not need to have certain kinds of constituents or mechanics to have emergent properties. It is important to emphasise that emergent properties are not mere epi-phenomena to systems; they feed back into the system and in themselves become a significant part of the operation of the system. Random patterns resulting from the interaction between components in a system do not make for emergent properties. Emergent properties are ordered and recurring patterns that come about through some kind of organisation among the components of that particular system. These recognisable structures are dynamic - they change over time. In other words, emergent phenomena are typically persistent patterns within a system with changing components. Furthermore, the possibilities of emergence are compounded when elements of the system allow for some capacity for adaptation and learning. ${ }^{26}$

The context in which an emergent pattern arises determines its function (Holland 1998: 121-226). In other words, environment plays a crucial part in the development of a system, in that a system's environment presents a number of constraints, which both curtail and enable the development of a certain structure. The resulting structure is the product of a complex interaction between the environment in which a system finds itself, the present state of the system and the history ${ }^{27}$ of the system. The boundaries between the system and its environment become difficult to determine because of this dynamic interaction.

Due to the dynamic character of the system, only certain aspects of the system can be analysed at a time. Hence, in order to facilitate analysis an artificial "frame" needs to be imposed on the system, which creates the possibility that any analysis could cause significant distortions in the system and implies that such a possibility should be taken into account in the final analysis. When we practise such framing our attention is attracted by a recurring pattern in a particular system and through a process of induction we construct a model of the selected phenomena. Only recurring patterns in a given system will be noticed and considered to be part of the mechanics of the system. Such a description of repeated elements will suggest rules or mechanisms according to which the system operates. It is the imposed frame that creates the safe space where there can be talk of truth, rationality, and identity. As long as we remain well within the frame our work can continue. Ambiguities arise at the limits of the discourse or frame.

Complex systems change continuously and in unpredictable ways. Such systems do

26 Some of the fundamental aspects of this kind of agent-based emergence are aptly captured in the metaphor of an ant colony. The individual agents (ants) have a limited repertoire, but the colony as a whole exhibits remarkable flexibility in its interaction with its environment and exhibits emergent behaviour which outstrips that of the individual agents (Holland 1998: 5).

27 "History" here refers to already actualised states of the system. A certain configuration of the system which is the result of previous states of the system and environmental constraints - already precludes some possible states, while enhancing the probability of the actualisation of others. History in this sense should not be understood as a chronological series of major events. The history of the system is contained in all the individual little interactions that take place all the time, distributed over the whole system. 
not operate according to the dictates of a telos; changes are unpredictable, irregular, and contingent. One of the most important characteristics of agent-based emergent behaviour is that there is no direction by a central executive agent or body (something analogous to Dennett's Cartesian Theatre). There are many advantages to not being directed by a single centre of control, but a system must, by definition, display coordinated and interactive behaviour. The idea in the complexity sciences is to explain how a complex system, like that of the biosphere or language, can attain such a high degree of order, without needing to postulate some external designing or directing agent or some form of executive internal control.

Self-organisation, as an emergent property of complex systems, explains how complex systems attain structure. Under pressure from its environment, the system seeks to enhance its cohesion and integration and functioning capabilities. In this manner a system "governs" itself.

In a self-organised system the individual components of the system react to information available to them locally, which translates into complex and organised behaviour on a systemic level. Any one node is not "aware" of the behaviour and structure of the entire system. The system itself relies on interactive behaviour and is subject to continuous change. Self-organising systems are also self-referential in that new components are "accepted" into the system by virtue of their ability to enhance the overall organisation of the system. The system's organisation makes for an internal selection process, established by the system itself, and operates to preserve and enhance the system. In other words, self-organising processes are primarily informational.

In the light of these characteristics of complex systems and their emergent properties we wish to argue that the self is an emergent property of a complex system, which comprises the mental apparatus, the rest of the body, the history of both the body and the environment. The self as an emergent property of this complex system would retain the narrative character of Dennett's theory, without falling into the pitfall of undermining the ousting of metaphysical homunculus from the mind/brain. Furthermore, we wish to argue that a complex approach to the narrative self could enhance Dennett's theory through suggesting the possible mechanisms in the brain-environment interaction that leads the human organism to taking up the intentional stance and hence creating a narrative about its own characteristics, functions and motivations. Further implications, which restore Freud to the discussion, are highlighted by a complex approach: namely, the role and importance of fantasy in creating a narrative self and the importance of an ability to generalise from experience as a defining characteristic of the human mind.

\section{The Emergent Self}

In the light of our discussion so far, the self can well be said to be an emergent property of a complex system of the genetic mental apparatus, the body, external influences (the environment - especially language) and their histories. If we concur with Dennett and Freud's analyses, we have the many components that are in interaction: genetic predisposition to construct a self, the mental apparatus itself - structured to learn and adapt - the body and its various states, memories of experiences, other people, language; the list goes on. It seems clear that these components are in interaction - the environment contributes to and influences the development of the mental apparatus, etc.

It seems entirely plausible that one's self could be very different given the possibility that one had made different choices, or had different influences and exposure to differ- 
ent circumstances than those that had actually been the case. All of these factors have to do with restrictions within the given system within which one constructs a self: physical ability, genetic predisposition, material circumstances, geographical location; the list is endless. It is also quite unproblematic to state that many of the possibilities that have been actualised, much of what has become part of one's self are, for all intents and purposes, irreversible.

Whereas one might agree that one's environment, genetic pre-dispositions, etc. all contribute to the structure of the self, one might still hold that these individual parts do not, in themselves indicate how a phenomenon like the self can possibly come to be. Hence the view that the mind, the self, or the soul is somehow mysterious and not of the world of matter.

Our argument would be that the phenomena of emergence and self-organisation, which we discussed as properties of complex systems, come into play at this point, and serve to preserve the materiality of the self. With these conceptual tools it becomes unnecessary to take recourse to inexplicable immaterial characteristics to account for the abstruseness of the self. The concept of self-organisation could also allow us to preserve Dennett's invaluable proposition of ridding consciousness of the Cartesian Theatre, while providing some theoretical underpinning to his bid.

As discussed in the previous section, novelties arise in complex systems that result from the system rules, but cannot be predicted from the properties of the components. These emergent properties are the characteristics of the system that result from the non- linear interactions between the components of the system amongst themselves, and with their environment; and are not mere epi-phenomena to systems, but become a significant part of the operation of the system.

If we agree with Freud that consciousness is the result of the structure of the mental apparatus and the need for the human organism to respond to external stimuli, and that much of the self consists of our memory traces; and with Dennett that consciousness and the self are products of the evolution of a specific species within a given environment, then the assertion that the self is an emergent property of a complex system is an entirely plausible proposition.

The interaction between the structures of the mental apparatus, its response to both internal and external stimuli, its capacity for memory, its ability to account for itself within its environment, and to plan and adapt within that environment, in terms of complexity theory, give rise to an additional property of the mental apparatus - the self. This additional property is wholly an effect of the structures of the mental apparatus and its environment, and as such is material. As discussed in the previous section, complex systems are dynamic and do not operate according to the dictates of a telos; changes are unpredictable, irregular, and contingent. Our material self develops and adapts in a specific structure, within a specific context, and does not possess a sort of Aristotelian formal cause or essence, other than the genetic predisposition to construct a self. This material quality need in no way detract from the portentousness of the self.

The self as an emergent property of a complex system cannot be thought of as an epi-phenomenon of that system. As we have discussed, an emergent property is not merely a spin-off from the workings of a certain system, but becomes part of the system in which it originates. Emergent properties feed back into their systems of origin, affect changes within those systems, and are again themselves influenced by such changes. As the self learns, develops and adapts, changes are brought about both within the mental apparatus and upon the environment; changes which cause the self 
to adapt yet again. Feedback takes place through various forms of language, and other artefacts.

Given the enormity of the possibilities open to the self, it begins to seem surprising that we seem to possess something like a coherent self, recognisable as belonging to the "same" person over time, at all. Or that, despite widely varying idiosyncrasies, all people can relatively safely be said to have a sense of self. How is it possible to account for a coherent self, recognisable as "the same" structure over time and through various circumstances and experiences? In fact, complexity theory provides us with a very handy way of accounting for such identity of self, while still allowing for profound changes to occur in the self, both diachronically and synchronically: self-organisation.

As we have seen, in a self-organised system the individual components of the system react to information available to them locally, which translates into complex and organised behaviour on a systemic level. The fundamentally relational character of complex systems ensures that the dynamics of the system itself provides the constraints within which changes at different levels and of varying magnitudes occur. We have seen that self-organising systems are self-referential in that the system's organisation makes for an internal selection process, established by the system itself, and operates to preserve and enhance the system. As argued the self, as an emergent property of a complex system, is imbedded in the system and, as such, is subject to the organisational constraints within the system. Thus the organisation and attributes of the system itself both originates and regulates the development of the self. Creating a narrative in which it depicts its own characteristics and motivations is a function of such self-referentiality in the human mind.

Freud believed that consciousness occurred when some "contents" of the mental apparatus were expressed verbally, or linked with language, and we have also seen that Dennett sees the self as a complex of memes. The emergent, self-organised self answers the criticism we had of Dennett's earlier conception, especially with regard to the apparent agency that Dennett's memes seem to posses. Language makes up an important part of the complex system in which the self arises. ${ }^{28}$ It both forms part of the environment in which the mental apparatus operates and provides information about the environment to the mental apparatus. One of the ways in which the mental apparatus feeds back into the environment is through language.

Language, and culture in general, makes up part of the system in which the self originates and functions and, as such, is also subject to systemic constraints. In this model, language/culture can contribute to the self and its development, without possessing any kind of agency or evolutionary motive. Human brains are not cultivated or infested by memes in order to ensure their (the memes') survival. By the same token, the self is not a "complex of memes". The self is a recurrent emergent pattern within a system, which includes language and culture, and hence is heavily influenced by language and culture, to the extent that these make up its environment. Typical to complex systems, the flow is not only one way, and the self feeds back into its environment, affecting changes within language and culture.

We concur with Dennett that the "self-like" patterns that emerge within the complex system of mental apparatus, language etc. are narrative in nature. Or, to be more precise, the mental picture that the mental apparatus factors into its model of the world in which it must operate is narrative in nature. As we have discussed, the possibilities

28 Language itself can be modelled as a complex system (see Cilliers 1998:37-47). 
within a complex system are vast. It is safe to assume that the "patterns of self" that arise within our complex system are numerous, diverse and varied and situated within a web of information that is largely unconscious. It is our contention that our mental apparatus operates in such a way as to "frame" the self within this dynamic web of patterns. This frame, as Dennett suggested, generally consists of taking up the intentional stance towards "itself". We have seen that, in working with complex systems, their very complexity requires that an artificial "frame" be imposed on the system, to create a space within which we can talk about identity, for example. We have also seen that, when we practise such framing, our attention is attracted by a recurring pattern in a particular system and through a process of induction we construct a model of the selected phenomena. The suggestion here is that a similar process takes place within the mental apparatus in which recurring patterns, which arise out of the dynamics of the system, are construed as "the self" of that system. It is important to stress that such "construction" is not enacted by some form of homunculus, but occurs purely as a function of the activities of the system as a whole. The form that the "definite" self takes on would be a narrative form, in that the mental apparatus would need to work with a model of the self that is coherent, comprehensible and consistent in terms of chronology and morphology. In other words, the system's model of itself needs to be applicable in both the diachronic and synchronic circumstances of the system. If this is the case, the system would need to be able to call upon events that have already occurred and the effects of these events in order to facilitate its factoring of itself into both its present and future situations; if this weren't possible we wouldn't be able to learn from experience. The system is in dire need of narratives; it learns how to construct narratives by being exposed to them through language.

A narrative presents events in terms of chronology and within a particular space. Usually events are presented as being related in one way or another and as having occurred in a particular sequence. Furthermore, a narrative usually creates a certain logical space within which relations are drawn and inferences are made. Within such a framework it becomes possible to draw conclusions and predict consequences, based on past experience.

Narrative is not only the mode in which our system models itself, it is also the mode in which the system presents itself to other similar systems (other people). Such a narrative is usually couched in terms of language. We present ourselves to others by relating stories about who we are. We create coherent pictures of our self and our motives - as if from a single source - in order to present ourselves to others in an accessible way.

Within this framework it also becomes possible to begin to account for the fact that people can share their experiences of "being" with others (what Dennett called heterophenomenology 1991). It is safe to assume that, due to our common genetic heritage, human beings generally have similar brain structures with relatively similar predispositions - one of which is to create a self. Furthermore, human beings usually interact with one another against a broadly common cultural backdrop, which means that they have at least some mind-tools, points of reference and experiences in common. These factors, in conjunction with our propensity to model the world in terms of a narrative (with the logical space that such a narrative circumscribes) should allow for the ability to access and interpret the narratives presented by other human beings in such a manner so as to allow understanding and insight into the experiences of others. 


\section{Conclusion}

We have argued that modelling the self as an emergent property of the complex system that consists of our mental apparatus, environment and language can enhance Dennett's theory of a narrative self, created by the human organism adopting the intentional stance towards itself. In this manner we are able to account for the self as an evolutionary and material entity.

In the previous section we suggested that presenting the self in the form of a narrative presents a logical space within which it becomes possible to adopt the intentional stance. This is because innumerable bits of information and processes in the brain need to be organised in some coherent manner in order to be a useful picture of the functions and motivations of the system. We suggested that the brain system learns how to compose a coherent narrative through being exposed to other narratives. Narratives, of course, find their manifestation in language (understood in its broadest sense).

Further advantages of taking a complexity theoretical approach to the self are the tensions that characterise different systems within the mental apparatus (as highlighted by Freud and touched upon by Dennett). It is our belief that complexity theory could play a significant role in exploring the way in which these tensions interact and compete in the formation of the self.

A further implication (again recalling the theories of Freud) highlighted by a complex approach, is the role and importance that fantasy might play in creating a narrative self. Perhaps fantasy may not only be utilised in simulating possible outcomes of actions (its function in Dennett's Popperian Creatures) but it might serve to smooth out or gloss over discrepancies in the narrative of self. Furthermore it may contribute significantly to the human mind's ability to generalise from experience. This ability to generalise from experience and the tremendous creativity that it allows may very well be the defining characteristic of the human mind and account for the gap between our mental capabilities and those of our closest relatives on the evolutionary tree. These are all implications that may be fruitfully developed in the future.

\section{References}

Cilliers, P. 1998. Complexity and Postmodernism: Understanding Complex Systems. Routledge, London and New York.

Cilliers, P. and Gouws, A. 2001: Freud's 'Project', Distributed Systems, and Solipsism, South African Journal of Philosophy, 2001, 20(3).

Dawkins, R. 1976: The Selfish Gene. Oxford: Oxford University Press.

Dennett, D.C. 1991. Consciousness Explained. London: Penguin.

Dennett, D.C. 1994 Beginnings and Sources, in A Companion to the Philosophy of Mind, Samuel Guttenplan (ed.), Oxford: Basil Blackwell Ltd.

Dennett, D.C. 1995: Darwin's Dangerous Idea. New York: Simon and Schuster.

Dennett. D.C. 1996: Kinds of Minds. London: Weidenfeld and Nicholson.

Descartes, R. 1978: A Discourse on Method, Meditations, and Principles. London: Everyman's Library.

Freud, S. 1901: The Interpretation of Dreams. Standard Edition, Volumes 4-5, London: Hogarth Press and the Institute of Psycho-Analysis. 
Freud, S. 1911: Formulations on the Two Principles of Mental Functioning. Standard Edition, volume 12, London: Hogarth Press and the Institute of Psycho-Analysis.

Freud, S. 1915a: Instincts and Their Vicissitudes. Standard Edition, volume 14, London: Hogarth Press and the Institute of Psycho-Analysis.

Freud, S. 1915b: The Unconscious. Standard Edition, volume 14, London: Hogarth Press and the Institute of Psycho-Analysis.

Freud, S. 1923: The Ego and the Id. Standard Edition, volume 19, London: Hogarth Press and the Institute of Psycho-Analysis.

Freud, S. 1925: A Note upon the "Mystic Writing-Pad." Standard Edition, volume 19, London: Hogarth Press and the Institute of Psycho-Analysis.

Freud, S. 1950 [1895]: Project for a Scientific Psychology. Standard Edition, volume 1, London: Hogarth Press and the Institute of Psycho-Analysis.

Gould, S. J. 2000: "More Things in Heaven and Earth" in Alas, Poor Darwin: Arguments Against Evolutionary Psychology. Rose, H and Rose, S (eds.), London: Jonathan Cape.

Heylighen, F. 1997: "Classic publications on complex, evolving systems: a citation-based survey", in: F. Heylighen, C. Joslyn and V. Turchin (eds.): Principia Cybernetica Web (Principia Cybernetica, Brussels), URL:

http://pespmc1.vub.ac.be/EVOCOPUB.html

Holland, J. 1998. Emergence: From Chaos to Order. Massachusetts: Helix Books.

Lucas, C. 2003: "Quantifying Complexity Theory”, in: C. Lucas (ed.): The Complexity and Artificial Life Research Concept for Self-Organising Systems, URL: $\mathrm{http} / /$ calresco.org/lucas/quantify.htm

Morowitz, H.J. 2002. The Emergence of Everything: how the world came to be complex. New York: Oxford University Press.

Nathan, P. W. 1998: Nervous System, in: The Oxford Companion to the Mind. R.L. Gregory (ed.). Oxford: Oxford University Press.

Strachey, 1986 (ed.) in: Freud, S. [1950]: Project for a Scientific Psychology. Standard Edition, volume 1, London: Hogarth Press and the Institute of Psycho-Analysis.

Sternberg, R.J. 1995: In Search of the Human Mind. Ford Worth: Harcourt Brace College Publishers.

Wikipedia Encyclopedia 2004: Computational complexity theory, in ...

URL: http://en2.wikipedia.org/wiki/Computational...complexity...theory

Zangwill, O. L. 1998: Freud on Mental Structure, in: The Oxford Companion to the Mind. R.L. Gregory (ed.). Oxford: Oxford University Press. 